\title{
Cost sharing solutions defined by non-negative eigenvectors
}

\author{
Begoña Subiza ${ }^{a, b}$, José A. Silva-Reus ${ }^{a, b}$ and Josep E. Peris ${ }^{a, b, c}$ \\ ${ }^{a}$ Departament de Mètodes Quantitatius i Teoria Econòmica. Universitat d'Alacant. \\ 03080 Alacant.Spain.E-mail adresses: subiza@ua.es, jose.silva@ua.es, peris@ua.es \\ ${ }^{b}$ Instituto Interuniversitario Desarrollo Social y Paz (IUDESP). \\ ${ }^{c}$ Corresponding author
}

\begin{abstract}
The problem of sharing a cost $M$ among $n$ individuals, identified by some characteristic $c_{i} \in \mathbb{R}_{+}$, appears in many real situations. Two important proposals on how to share the cost are the egalitarian and the proportional solutions. In different situations a combination of both distributions provides an interesting approach to the cost sharing problem. In this paper we obtain a family of (compromise) solutions associated to the Perron' eigenvectors of Levinger's transformations of a characteristics matrix $A$. This family includes both the egalitarian and proportional solutions, as well as a set of suitable intermediate proposals, which we analyze in some specific contexts, as claims problems and inventory cost games.
\end{abstract}

Keywords: Cost Sharing, Egalitarian, Proportional, Perron's Eigenvector, Compromise Solution

\section{Introduction}

A (cost-surplus) sharing problem consists of the division of a certain amount $M$ among a group of $n$ agents. We assume that each agent $i$ $(i=1,2, \ldots, n)$ is identified by means of a (numerical) characteristic $c_{i}$ relevant in the distribution of the total amount $M$. We may find many real situations covered by this simple model. Let us observe, for instance, the following ones:

[ 1 ] A typical example is how to allocate the total cost $M$ of a facility (for instance, a water supply system) among the $n$ towns that will share 
it. The characteristic $c_{i}$ may be, in this example, the proportion of the total population living in each of these towns. A cost allocation is a vector $x=\left(x_{1}, x_{2}, \ldots, x_{n}\right)$ such that $x_{1}+x_{2}+\ldots+x_{n}=M$. The two more popular ways to share the total cost $M$ are the egalitarian and the proportional ones (see, for instance, Moulin (1988)).

[2] Another example comes from analyzing costs in one-to-many distribution systems. This kind of problem has a logistic feature, namely the distribution cost (see, for instance, Turkensteen and Klose (2012)). A single facility, whose location is yet to be determined, serves geographically dispersed demand points (consumers). Once the location of the facility and the distribution cost (the cost of the optimal delivery route) $M$ has been obtained, the problem to be analyzed is how this cost $M$ is allocated to consumers. Again, there are two focal positions: the cost is allocated in an egalitarian way, so any costumer pays (per unit) an equal part of the total distribution cost; or, the cost is allocated in a proportional way with respect to the characteristic $c_{i}$, that in this example could represent the distance between the facility and the consumer $i$, or the cost of a direct delivery from the facility to the consumer.

[ 3 ] The so-called claims problem (O'Neill, 1982) involves $n$ agents identified by some claims $c_{i}, i=1,2, \ldots, n$ on a estate $M$ that is not sufficient to cover the aggregate claim $C=\sum_{i=1}^{n} c_{i}$, that is $C>M$. The problem is how to allocate the estate $M$ among the claimants by taking into account the claims $c_{i}$ the agents have on it. Among the many rules defined in this class of problems, two prominent solutions are the proportional and the egalitarian ones.

[ 4 ] In a recent paper, Karsten and Basten (2014) present a model on how to share the benefits in pooling of spare parts between multiple users. As they mention, "important savings may be obtained if pooling is taken into account. While promising this does raise the question on how the [users] should share the benefits". In order to construct their model, they use the proportional rule to divide the costs (in this case, proportionality is with respect to the demand rate of each individual). Instead of using proportionality, an egalitarian allocation could be also considered. A similar situation may be found in Meca et al. (2004) (see also Fiestras-Janeiro et al. (2011)) where an inventory cost game 
is presented in order to allocate the benefits of cooperation. They use a proportional approach in order to define a solution that is proved to be on the core of the cooperative game.

All of the above situations may be expressed by the abstract model:

An amount $M$ must be shared among agents $i=1,2, \ldots, n$ by considering the individuals' characteristics $c_{i}$.

As mentioned, the proportional and the egalitarian solutions are two focal approaches to solve this model. In many situations, a combination of these solutions could make sense. For instance, if we go back to the water supply system example, it is clear that the population (the characteristic) of each town affects the size of the system. Then, a proportional (with respect to the characteristics) distribution of the cost seems reasonable. But, on the other hand, most of the cost doesn't depend on the supplied population, so an egalitarian share (regardless of the characteristics) could be as reasonable as the proportional division. As consequence, a combination of both distributions provides an interesting approach. Our proposal is within this perspective.

One possibility is to consider convex combinations of these two solutions. This idea has been recently developed in the context of claims problems (situation [3]) where the characteristic $c_{i}$ represents the individual $i^{\prime}$ s claim (see Giménez-Gómez and Peris (2014)). In that article they propose the $\alpha_{\text {min }}$ solution by using a specific convex combination of the egalitarian and the proportional solutions with the idea of guaranteeing a minimal amount to each individual (regardless of their claims) and allocating the remaining in a proportional way.

We propose an alternative approach to obtain intermediate solutions: instead of taking convex combinations of the egalitarian and proportional solutions, we use eigenvectors of some suitable matrices. In so doing, we define the characteristics matrix of a problem as the matrix whose rows are all identical to the vector of individuals' characteristics $c_{i}$. By using this matrix $A$, we get an alternative way of obtaining the main solutions. In particular, the egalitarian solution corresponds to the right eigenvector of matrix $A$ and the proportional solution corresponds to the left eigenvector (or, equivalently, to the right eigenvector of the transpose matrix $A^{t}$ ). Then, we present our family of compromise solutions for the sharing problem, as 
the ones associated to the Perron's eigenvectors of each convex combination of the characteristics' matrix and its transpose (Levinger's transformation). The egalitarian and proportional solutions are obtained for $\alpha=0$ and $\alpha=1$, respectively. We analyze the main properties of these compromise solutions as functions of the parameter $\alpha$ that defines the convex combination.

When analyzing some particular situations, we realize that the egalitarian solution could propose inadmissible allocations. For instance, in situation $[2]$, if $c_{i}$ represents the cost of the individual delivery, rationality implies that no agent should pay more than this amount. In situation [3] the characteristic $c_{i}$ denotes the claim that individual $i$ has on the endowment. Then, it is obvious that no agent should receive more than her claim. Finally, in situation [4] it is important the proposal to give an allocation in the core of the cooperative game. As in the egalitarian case, other solutions associated to Perron's eigenvectors of Levinger's transformations could be inadmissible proposals. However, we will show that it is always possible to find some admissible ones.

The paper is organized as follows. Section 2 introduces the mathematical model we are going to use, and defines the characteristics matrix associated to a cost sharing problem. In Section 3 we obtain the main properties of eigenvalues and eigenvectors of the convex combinations of the characteristics matrix and its transpose. Finally, in Section 4, we analyze some particular situations (claims problems and inventory cost games).

\section{Main definitions}

\subsection{Cost sharing problems}

A cost sharing problem with relevant characteristics is defined by a finite set of agents $N=\{1,2, \ldots, n\}$, the total cost $M$ to be allocated to the agents, and the characteristics vector ${ }^{1} c^{t}=\left[c_{1}, c_{2}, \ldots, c_{n}\right]$, identifying each agent. The issue is how to share the total cost $M$ among the agents. ${ }^{2}$

A cost sharing solution (sharing rule) associates to each sharing problem $(M, c)$, an allocation $x=\left(x_{1}, x_{2}, \ldots, x_{n}\right) \in \mathbb{R}^{n}$, such that $x_{i} \geq 0$ (no subsidies

\footnotetext{
${ }^{1}$ Throughout the paper, vectors are considered column vectors, $v_{n \times 1}$. The transposed (row) vector is denoted by $v^{t}$.

2 Although we consider cost sharing problems, our reasoning could be immediately applied to surplus sharing situations.
} 
are allowed) and $\sum_{i=1}^{n} x_{i}=M$ (efficiency). The value $x_{i}$ is the amount individual $i$ contributes to the total cost $M$.

The most popular sharing rules are the egalitarian (Egal) and the proportional (Prop) ones. Equal division establishes that each agent is allocated the same part of the total amount,

$$
x_{i}=\operatorname{Egal}_{i} \equiv \frac{M}{n}, \text { for all } i \in N .
$$

Proportional division, with respect to vector $c$ establishes the cost allocation

$$
x_{i}=\operatorname{Prop}_{i} \equiv \frac{c_{i}}{\sum_{i=1}^{n} c_{i}} M, \text { for all } i \in N .
$$

If we denote by $v_{e g}$ the vector with all entries equal to $1 / n$, and by $v_{p r}$ the vector whose $i$-component is $\frac{c_{i}}{\sum_{i=1}^{n} c_{i}}$, then the egalitarian and proportional solutions may be written as:

$$
\text { Egal }=M v_{e g}, \quad \operatorname{Prop}=M v_{p r} .
$$

\subsection{Matrix analysis}

Given a square positive matrix $A\left(a_{i j}>0\right.$, for all $\left.i, j\right)$, Perron's theorem (Perron, 1907) ensures the existence of an eigenvalue of $A, \lambda(A)$, which is strictly positive and has associated a positive eigenvector $v^{*}>0$ such that $A v^{*}=\lambda(A) v^{*}$, and $\lambda(A)=\rho(A)$, the spectral radius of this matrix. ${ }^{3}$ We consider positive eigenvectors normalized so that the sum of its components is equal to $1, \sum_{i=1}^{n} v_{i}^{*}=1$. Note that this fact guarantees the uniqueness of the associated positive eigenvector.

Given a positive square matrix $A$, the Levinger's transformation of $A$ (Levinger, 1970) is the family of matrices obtained throughout convex combinations of $A$ and its transpose $A^{t}$, that is, defined by

$$
\mathcal{A}(\alpha)=\alpha A^{t}+(1-\alpha) A \quad \alpha \in[0,1]
$$

We denote by $v(\alpha)$ the normalized positive eigenvector associated with the dominant eigenvalue $\lambda(\alpha)$, in the corresponding $\alpha$ Levinger's transformation.

\footnotetext{
${ }^{3}$ Moreover, this eigenvalue is simple, strictly greater than any other eigenvalue, and no other eigenvalue has a positive eigenvector associated. See, for instance, Berman and Plemmons (1994).
} 
We know (see Fiedler (1995)) that the Levinger's function, defined by Perron's eigenvalue $\lambda(\alpha)$, is a non-decreasing function in the sub-interval $\left[0, \frac{1}{2}\right]$ and that it is symmetric about $\alpha=\frac{1}{2}$.

\subsection{From eigenvectors to sharing proposals}

Any positive normalized vector $v^{*}>0$ provides a way of sharing an amount $M$ among $n$ individuals:

$$
x_{i}=M v_{i}^{*}, \quad i=1,2, \ldots, n \text {. }
$$

If we use the normalized eigenvectors $v(\alpha)$, associated to the Levinger's transformation $\mathcal{A}(\alpha)$, then we obtain a family of possible cost allocations, by varying the parameter $\alpha$,

$$
x_{i}(\alpha)=M v_{i}(\alpha), \quad i=1,2, \ldots, n .
$$

\subsection{A matrix associated to a cost sharing problem}

We are not interested in general positive matrices, but in a particular class defined by the characteristics of the individuals involved in the cost sharing problem. In order to introduce our matrix, let us consider a cost sharing problem $(M, c)$ and define the square matrix $A$ associated to this problem by:

$$
A=e c^{t}=\left(\begin{array}{cccc}
c_{1} & c_{2} & \ldots & c_{n} \\
c_{1} & c_{2} & \ldots & c_{n} \\
\ldots & \ldots & \ldots & \ldots \\
c_{1} & c_{2} & \ldots & c_{n}
\end{array}\right)
$$

where $e^{t}=[1,1, \ldots, 1]$. Without loss of generality, we consider the components in vector $c$ increasingly ordered:

$$
c_{1} \leq c_{2} \leq \ldots \leq c_{n}
$$

and normalized (taken in relative sizes), so that $\sum_{i=1}^{n} c_{i}=1$. It must be noticed that the (normalized) eigenvector associated to a matrix $B$ coincides with the one associated to any proportional matrix $A=\gamma B$, for any $\gamma \in \mathbb{R}$. This fact allows us to select the matrix associated to a cost sharing problem with row sum equals to 1. 
Due to the normalization of vector $c$, matrix $A$ is stochastic ${ }^{4}$ and it is immediate to observe that

$$
\lambda(A)=1, \quad A v_{e g}=v_{e g}, \quad A^{t} v_{p r}=v_{p r} .
$$

Then, by using our notation as Levinger's normalized eigenvectors,

$$
\text { Egal }=M v(0), \quad \text { Prop }=M v(1)
$$

Moreover, given these allocations, it is clear that any convex combination of the eigenvectors, $v=\alpha v_{p r}+(1-\alpha) v_{e g}=\alpha v(1)+(1-\alpha) v(0)$ defines a new distribution that provides an intermediate proposal among those.

\subsection{Compromise solutions}

An alternative approach to obtaining intermediate (compromise) proposals could be to compute convex combinations of the matrices $A$ and $A^{t}$ and proposing the Perron's eigenvector of this convex combination as the way of defining the new allocation. In other words, we want to use the Perron's normalized eigenvector associated to Levinger's transformation of the characteristics matrix $A, \mathcal{A}(\alpha)=\alpha A^{t}+(1-\alpha) A$. For each $\alpha$ the eigenvector $v(\alpha)$ defines a solution $x(\alpha)=M v(\alpha)$ to the cost sharing problem $(M, c)$. We are interested in analyzing the properties of these allocations as functions of the parameter $\alpha$ that defines the transformation. First, we show that $\lambda(\alpha)$ is a symmetric function that achieves its maximum at $\alpha=\frac{1}{2}$, and moreover we will prove that it is a concave function. However, our main interest is the analysis of the behaviour of the normalized components of the positive associated eigenvectors $v(\alpha)$ as non linear functions of the parameter $\alpha$, since these components provide the compromise solution.

Definition 1. Given a cost sharing problem with total cost $M$ and individuals' characteristics $c_{1} \leq c_{2} \leq \ldots \leq c_{n}$, the compromise solution associated to $\alpha \in[0,1]$ is the allocation defined by

$$
x(\alpha) \equiv M v(\alpha) .
$$

\footnotetext{
${ }^{4}$ A stochastic matrix (also termed probability matrix, or Markov matrix) is a square matrix such that each of its entries is a non-negative real number representing a probability (so, each row sum is 1). See, for instance, Berman and Plemmons (1994) for formal definitions and main properties of stochastic matrices.
} 
The following example compares the distributions proposed by the egalitarian and the proportional solutions and illustrates our compromise solutions.

Example 1. Consider the cost sharing problem of a common facility (a water supply system) that will be used by five cities. We suppose that the total cost is $M=100$ (consider percentage) and that the characteristics $d_{i}$ are identified by the population (in thousands) living in each city, that we suppose $\left[d_{1}=200, d_{2}=240, d_{3}=380, d_{4}=460, d_{5}=720\right]$. Then, the egalitarian solution gives the cost sharing Egal $=(20,20,20,20,20)$. The proportional (with respect to the population in each city) solution proposes the cost sharing Prop $=(10,12,19,23,36)$.

The population in each city (normalized so that the total sum of the population is equal to 1$)$ is $c^{t}=[0.10,0.12,0.19,0.23,0.36]$. Then, the characteristics matrix is

$$
A=\left(\begin{array}{lllll}
0.10 & 0.12 & 0.19 & 0.23 & 0.36 \\
0.10 & 0.12 & 0.19 & 0.23 & 0.36 \\
0.10 & 0.12 & 0.19 & 0.23 & 0.36 \\
0.10 & 0.12 & 0.19 & 0.23 & 0.36 \\
0.10 & 0.12 & 0.19 & 0.23 & 0.36
\end{array}\right)
$$

If we now consider the convex combination given by $\alpha=0.3282$, the Perron's eigenvalue is $\lambda(\alpha)=1.0453$ and the cost allocation defined by the Perron's eigenvector is $x(\alpha)=(16.86,17.49,19.69,20.94,25.02)$, which is a compromise between the egalitarian and the proportional approaches. Note that this result is different from the one we obtain by considering the convex combination (for $\alpha=0.3282$ ) of the egalitarian and proportional solutions: $\alpha$ Prop $+(1-\alpha) E g a l=(16.72,17.37,19.67,20.98,25.25)$. In fact, there is not $\alpha \in[0,1]$ such that the proposal given by $x(\alpha)$ could be obtained as a direct convex combination of the egalitarian and the proportional solutions.

\section{Main properties of the compromise solutions}

In Theorem 1 we obtain the expression of the dominant eigenvalue $\lambda(\alpha)$ and its main properties. Part of this result is a particular case of a result stated without proof by Levinger (see Levinger (1970) and Fiedler (1995)). We provide an elemental proof. 
Theorem 1. Let $A$ be a characteristics matrix. Then, the Levinger's function $\lambda:[0,1] \rightarrow \mathbb{R}$, defined by $\lambda(\alpha)=\rho(\mathcal{A}(\alpha))$, is continuous, symmetric around $\alpha=\frac{1}{2}$ and strictly concave in the interval $[0,1]$, achieving its maximum value at $\alpha=\frac{1}{2}$ and its minimum at $\alpha=0$, and $\alpha=1$, where $\lambda(0)=\lambda(1)=1$.

Proof. It is easy to check that, for all $\alpha \in(0,1), r k(\mathcal{A}(\alpha))=2$, and then the characteristic polynomial of this matrix is:

$$
p_{\mathcal{A}(\alpha)}(\lambda)=(-\lambda)^{n-2}\left((-\lambda)^{2}+p_{1}(-\lambda)+p_{2}\right),
$$

where $p_{1}$ and $p_{2}$ are the order 1 and order 2 trace of matrix $\mathcal{A}(\alpha)$,

$$
p_{1}=\sum_{i=1}^{n} c_{i}=1, \quad p_{2}=\alpha(1-\alpha) \sum_{i<j}\left(c_{i}-c_{j}\right)^{2} .
$$

Then,

$$
\lambda(\alpha)=\frac{1+\sqrt{1+4 \alpha(1-\alpha) \sum_{i<j}\left(c_{i}-c_{j}\right)^{2}}}{2} .
$$

We can immediately observe that this function is continuous, symmetric around $\alpha=\frac{1}{2}$, strictly concave, minimizes at $\alpha=0$ and $\alpha=1$, and maximizes at $\alpha=\frac{1}{2}$. Moreover, the greatest value of $\lambda(\alpha)$ depends on the value $B=$ $\sum_{i<j}\left(c_{i}-c_{j}\right)^{2}$ that can be seen as a "certain degree of variance" of the components of the characteristics vector $c: \lambda\left(\frac{1}{2}\right)=\frac{1+\sqrt{1+B}}{2}$.

Example 2. Consider the matrix A defined by individuals' characteristics vector $c^{t}=[0.05,0.1,0.15,0.25,0.45]$. Figures 1,2 and 3 show, respectively, the graph of $\lambda(\alpha)$, the graph of the components of the positive normalized eigenvector associated to $\lambda(\alpha), v_{i}(\alpha)$, and the graph of their second derivative, $v_{i}^{\prime \prime}(\alpha)$, for $\alpha \in[0,1]$ and $i=1,2,3,4,5$.

As we can observe, the eigenvalue function is as indicated in Theorem 1. On the other hand, the components of the eigenvector functions do not intersect and have a monotone behaviour (some of them increasing and the remaining ones decreasing). More interesting is the behaviour of the second derivative of the eigenvector functions: each component changes from concave to convex, or vice-versa, but all of them do this change at the same value of $\alpha$. We will prove later that these properties are true in general (see Theorems 3 and 4$)$. 


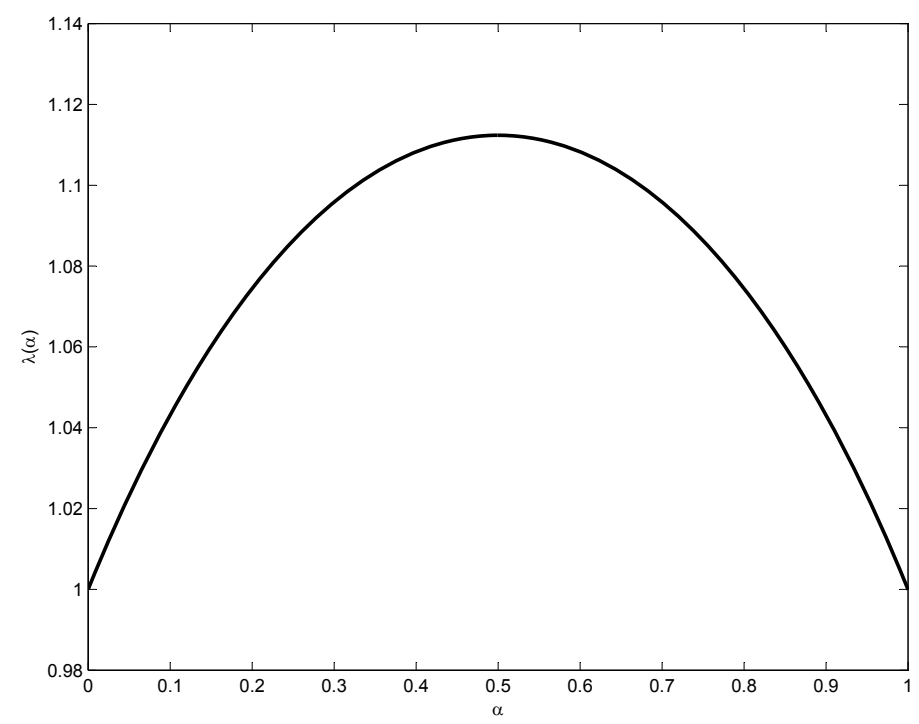

Figure 1: $\lambda(\alpha)$ as a function of $\alpha$ in Example 2.

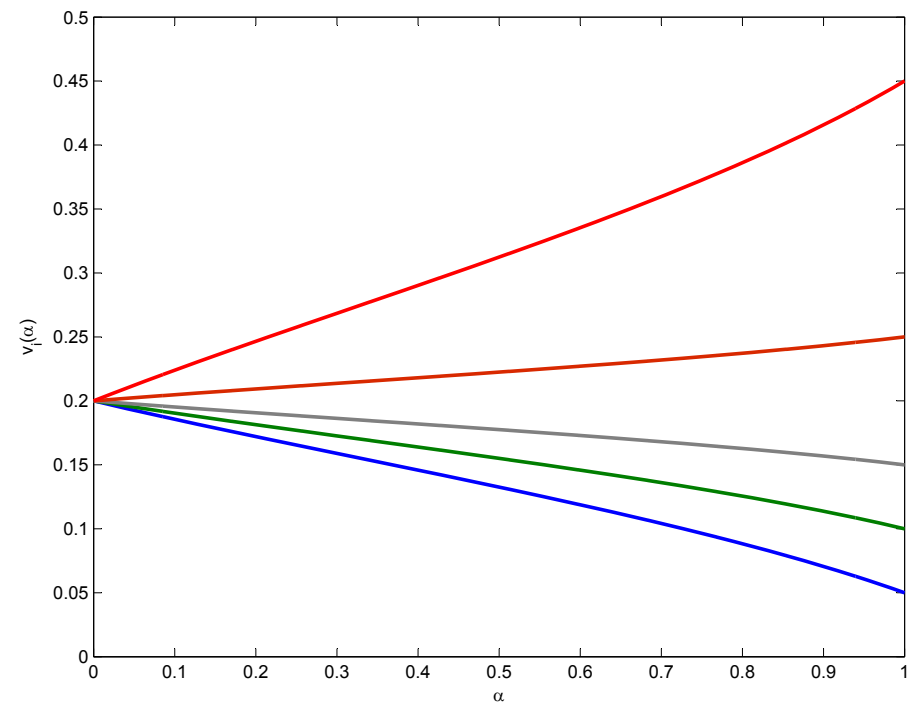

Figure 2: $v_{1}(\alpha), v_{2}(\alpha), v_{3}(\alpha), v_{4}(\alpha), v_{5}(\alpha)$, from bottom to top in Example 2. 


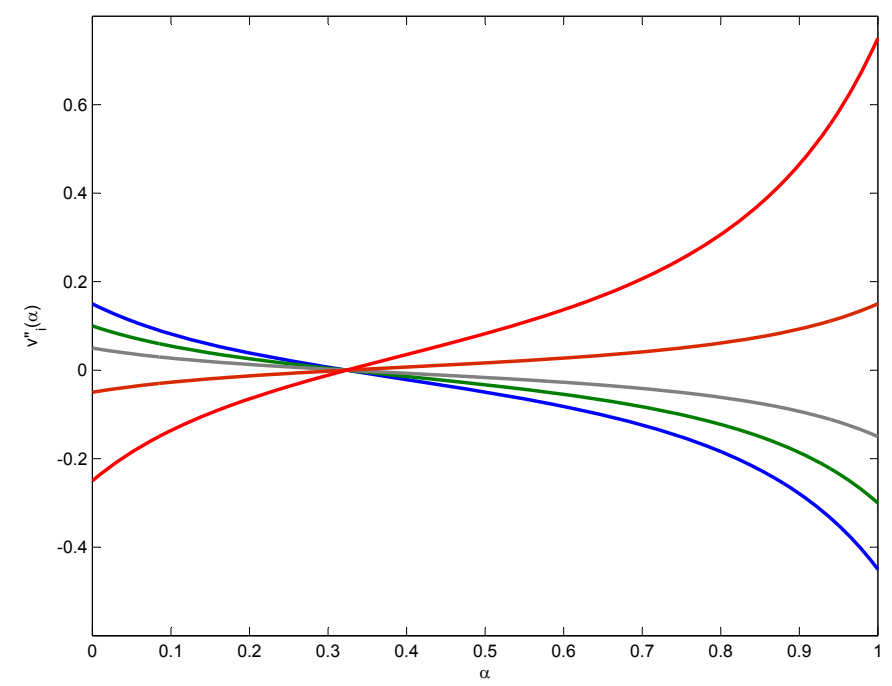

Figure 3: $v_{1}^{\prime \prime}(\alpha), v_{2}^{\prime \prime}(\alpha), v_{3}^{\prime \prime}(\alpha), v_{4}^{\prime \prime}(\alpha), v_{5}^{\prime \prime}(\alpha)$ in Example 2.

In the following result we show that, for a fixed $\alpha$, the components of the positive eigenvector $v(\alpha)$ are increasingly ordered. This fact is useful in their application to distribution problems, since the characteristics $c_{i}$ often represent some rights or duties and then a desirable property is that more rights (duties) deserve more revenues (payments), which are given by $v_{i}(\alpha)$.

Theorem 2. Let $A$ be a characteristics matrix and $v(\alpha)$ the normalized Perron's eigenvector associated to the Levinger's transformation $\mathcal{A}(\alpha)=$ $\alpha A^{t}+(1-\alpha) A$. Then, $v_{i}(\alpha) \leq v_{i+1}(\alpha)$, for all $i=1,2, \ldots, n-1$ and all $\alpha \in[0,1]$

Proof. The proof is based on the fact that we can compute the positive eigenvector $v(\alpha)$ by using the power method (see, for instance, Berman and Plemmons (1994))

$$
v(\alpha)=\lim _{k \rightarrow \infty} \frac{\mathcal{A}^{k}(\alpha) e}{e^{t} \mathcal{A}^{k}(\alpha) e} .
$$

Then, it is enough to observe that, for any $k, \mathcal{A}^{k}(\alpha) e$ is a conic combination of vectors $e$ and $c$ and then it preserves the order provided by $c$, independently of the value of $\alpha \in[0,1]$. 
As an immediate consequence, we obtain that our compromise solutions satisfy the (desirable) property of characteristic monotonicity. When applied to claims problems (situation [3]) it means that greater claims obtain greater allocations. In cost sharing problems (situations [1], [2], [4]) greater demands imply greater cost.

Corollary 1. Let us consider a cost sharing problem $(M, c)$. Then, the compromise solutions $x(\alpha)$ are characteristic monotonic:

$$
c_{i} \leq c_{j} \quad \Rightarrow x_{i}(\alpha) \leq x_{j}(\alpha) \quad \forall \alpha \in[0,1] .
$$

Our next result analyzes the behaviour of functions $v_{i}(\alpha)$ and shows how they increase or decrease, by depending on the value of $c_{i}$.

Theorem 3. Let $A$ be a characteristics matrix and $v(\alpha)$ the normalized Perron's eigenvector associated to the Levinger's transformation $\mathcal{A}(\alpha)=$ $\alpha A^{t}+(1-\alpha) A$. Let us suppose $c \neq \frac{1}{n} e$. Then,

a) $v_{i}(\alpha)$ is a decreasing non linear function in the interval $[0,1]$ for all $i$ such that $c_{i}<\frac{1}{n}$

b) $v_{i}(\alpha)$ is an increasing non linear function in the interval $[0,1]$ for all $i$ such that $c_{i}>\frac{1}{n}$

c) $v_{k}(\alpha)$ is a constant function in the interval $[0,1]$ if $c_{k}=\frac{1}{n}$

In order to prove Theorem 3 we will use the following Lemma. Let us denote by $e^{k}$ the $k$-th vector in the canonical basis, $e_{k}^{k}=1, e_{j}^{k}=0$, for each $j \neq k$.

Lemma 1. Let $V$ the space generated by vectors e and $c, V=\langle e, c\rangle$. Then,

a) $V^{\perp}=\left\{w \in \mathbb{R}^{n} \mid w^{t} \mathcal{A}(\alpha)=0\right\}$ for all $\alpha \in(0,1)$.

b) $v(\alpha) \in V$ for all $\alpha \in[0,1]$.

c) $e^{k} \in\left\langle V^{\perp}, e\right\rangle \Leftrightarrow c_{k}=\frac{1}{n}$. 
Proof. In order to prove part $a$ ) note that

$$
\mathcal{A}(\alpha)=\alpha c e^{t}+(1-\alpha) e c^{t},
$$

so every vector $w \in V^{\perp}$ fulfills $w^{t} \mathcal{A}(\alpha)=0$. As the dimensions of both vector spaces coincide (note that $r k(\mathcal{A}(\alpha))=2$, for $\alpha \in(0,1)$ ) we obtain the result.

b) Let $w \in V^{\perp}, w \neq 0, \alpha \in(0,1)$ and $v(\alpha)$ the positive eigenvector associated to $\lambda(\alpha)$. From part $a)$,

$$
w^{t} \lambda(\alpha) v(\alpha)=w^{t} \mathcal{A}(\alpha) v(\alpha)=0 .
$$

As $\lambda(\alpha) \neq 0, v(\alpha) \in\left(V^{\perp}\right)^{\perp}=V$.

c) Let us consider $\left\{w^{1}, w^{2}, \ldots, w^{n-2}\right\}$ a basis of $V^{\perp}$ and suppose that there exist $\mu, \mu_{1}, \mu_{2}, \ldots, \mu_{n-2} \in \mathbb{R}$ such that

$$
e^{k}=\mu e+\mu_{1} w^{1}+\mu_{2} w^{2}+\ldots+\mu_{n-2} w^{n-2} .
$$

Then,

$$
\begin{gathered}
\left(e^{k}\right)^{t} \mathcal{A}(\alpha)=\mu e^{t} \mathcal{A}(\alpha)+\mu_{1}\left(w^{1}\right)^{t} \mathcal{A}(\alpha)+\ldots+\mu_{n-2}\left(w^{n-2}\right)^{t} \mathcal{A}(\alpha)= \\
=\mu e^{t} \mathcal{A}(\alpha)=\mu \alpha e^{t}+\mu(1-\alpha) n c^{t} .
\end{gathered}
$$

On the other hand,

$$
\left(e^{k}\right)^{t} \mathcal{A}(\alpha)=\alpha c_{k} e^{t}+(1-\alpha) c^{t} .
$$

From both equalities, we obtain,

$$
(1-n \mu)(1-\alpha) c^{t}=\alpha\left(\mu-c_{k}\right) e^{t},
$$

for all $\alpha \in(0,1)$; then

$$
1-n \mu=0 ; \quad \mu-c_{k}=0 \quad \Rightarrow \quad c_{k}=\frac{1}{n} .
$$

Conversely, if $c_{k}=\frac{1}{n}$, by taking $\mu=\frac{1}{n}$, it is easy to observe that

$$
\left(e^{k}-\mu e\right)^{t} \mathcal{A}\left(\frac{1}{2}\right)=0 .
$$

That is, $\left(e^{k}-\mu e\right) \in V^{\perp}$, so $e^{k} \in\left\langle V^{\perp}, e\right\rangle$.

\section{Proof of Theorem 3.}

Parts $a$ ) and $b$ ): since $v_{i}(0)=\frac{1}{n}$, and $v_{i}(1)=c_{i}$, it is enough to prove that for all $i$ such that $c_{i} \neq \frac{1}{n}$, then $v_{i}^{\prime}(\alpha) \neq 0, \forall \alpha \in(0,1)$. If there is some $i$, and some $\alpha^{*} \in(0,1)$ such that $v_{i}^{\prime}\left(\alpha^{*}\right)=0$, then we have: 
[ 1 ] $\left(e^{i}\right)^{t} v^{\prime}\left(\alpha^{*}\right)=v_{i}^{\prime}\left(\alpha^{*}\right)=0$

[2] $e^{t} v^{\prime}\left(\alpha^{*}\right)=0$, since $e^{t} v\left(\alpha^{*}\right)=1$

[ 3$]\left(w^{j}\right)^{t} v^{\prime}\left(\alpha^{*}\right)=0, \forall j=1,2, \ldots, n-2$, as a consequence of Lemma 1

As $r k\left(e^{i}, e, w^{j}, j=1,2, \ldots, n-2\right)=n$, the linear system

$$
\begin{aligned}
& {[1]\left(e^{i}\right)^{t} x=0,} \\
& {[2] e^{t} x=0} \\
& {[3]\left(w^{j}\right)^{t} x=0, \forall j=1,2, \ldots, n-2,}
\end{aligned}
$$

has a unique solution $x=0$, so $v^{\prime}\left(\alpha^{*}\right)=0$. We are going to show that this fact is not possible. If we compute

$$
\begin{gathered}
(v(\alpha))^{t} \mathcal{A}(\alpha) v(\alpha)=\lambda(\alpha) \sum_{i=1}^{n} v_{i}(\alpha)^{2} \\
(v(\alpha))^{t} \mathcal{A}(\alpha) v(\alpha)=(v(\alpha))^{t}\left(A+\alpha\left(A^{t}-A\right)\right) v(\alpha)=\sum_{i=1}^{n} c_{i} v_{i}(\alpha)
\end{gathered}
$$

Equalizing both terms, and evaluating the derivatives at $\alpha=\alpha^{*}$ :

$$
\sum_{i=1}^{n} c_{i} v_{i}^{\prime}\left(\alpha^{*}\right)=\lambda^{\prime}\left(\alpha^{*}\right) \sum_{i=1}^{n} v_{i}\left(\alpha^{*}\right)^{2}+\lambda\left(\alpha^{*}\right) \sum_{i=1}^{n} 2 v_{i}\left(\alpha^{*}\right) v_{i}^{\prime}\left(\alpha^{*}\right)
$$

As $v^{\prime}\left(\alpha^{*}\right)=0$, and $v\left(\alpha^{*}\right) \neq 0$, then $\lambda^{\prime}\left(\alpha^{*}\right)=0$, so $\alpha^{*}=\frac{1}{2}$. On the other hand, by computing $c^{t} \mathcal{A}(\alpha) v(\alpha)$, with a similar argument we obtain that

$$
\sum_{i=1}^{n} c_{i} v_{i}(\alpha)=\frac{\alpha \sum_{i=1}^{n} c_{i}^{2}}{\lambda(\alpha)-1+\alpha}
$$

By deriving and substituting at $\alpha^{*}=\frac{1}{2}$,

$$
0=\left(\sum_{i=1}^{n} c_{i}^{2}\right) \frac{\lambda\left(\frac{1}{2}\right)-1}{\left(\lambda\left(\frac{1}{2}\right)-\frac{1}{2}\right)^{2}}
$$

which is not possible. 
Part c): Lemma 1 establishes the equivalence between conditions $c_{k}=\frac{1}{n}$ and $e^{k}=\frac{1}{n} e+\sum_{j=1}^{n-2} \mu_{j} w^{j}$. Then,

$$
\left(e^{k}\right)^{t} \mathcal{A}(\alpha) v(\alpha)=\lambda(\alpha)\left(e^{k}\right)^{t} v(\alpha)=\lambda(\alpha) v_{k}(\alpha)
$$

and

$$
\begin{aligned}
\left(e^{k}\right)^{t} \mathcal{A}(\alpha) v(\alpha) & =\frac{1}{n} e^{t} \mathcal{A}(\alpha) v(\alpha)+\sum_{j=1}^{n-2} \mu_{j}\left(w^{j}\right)^{t} \mathcal{A}(\alpha) v(\alpha)= \\
& =\frac{1}{n} \lambda(\alpha) \sum_{i=1}^{n} v_{i}(\alpha)=\frac{1}{n} \lambda(\alpha)
\end{aligned}
$$

As $\lambda(\alpha) \neq 0$, we obtain $v_{k}(\alpha)=\frac{1}{n}$, for all $\alpha \in(0,1)$.

Remark 1. The case not covered in this result, $c=\frac{1}{n} e$, is obvious since then matrix $A$ is a scalar matrix, $a_{i j}=\frac{1}{n}$ for all $i, j=1,2, \ldots, n$. In this case $\mathcal{A}(\alpha)=A$ for all $\alpha \in[0,1]$. Then the eigenvector is constant, $v(\alpha)=\frac{1}{n}$ e for all $\alpha \in[0,1]$.

In the case of a cost sharing problem, the above result splits the set of agents into two groups: those who have characteristics greater than the average $\left(c_{i}>\frac{1}{n}\right)$ and those under it. For the first ones, function $v_{i}(\alpha)$ (the amount they pay) is increasing with $\alpha$, whereas for the rest of agents it is decreasing.

Our last result analyzes the concavity/convexity of the $v_{i}(\alpha)$ functions. The most interesting fact is that all of them change from concave to convex (or vice-versa) at the same point, which is near to $\frac{1}{3}$ independently of the number of individuals.

Theorem 4. Let $A$ be a characteristics matrix and $v(\alpha)$ the normalized Perron's eigenvector associated to the Levinger's transformation $\mathcal{A}(\alpha)=$ $\alpha A^{t}+(1-\alpha) A$. Let us suppose $c \neq \frac{1}{n}$ e. Then, there is $\alpha_{g} \in(0,1)$ such that for all $i=1,2, \ldots, n$,

a) $v_{i}(\alpha)$ is a convex function in the interval $\left[0, \alpha_{g}\right]$ and a concave function in the interval $\left[\alpha_{g}, 1\right]$, if $c_{i}<\frac{1}{n}$ 
b) $v_{i}(\alpha)$ is a concave function in the interval $\left[0, \alpha_{g}\right]$ and a convex function in the interval $\left[\alpha_{g}, 1\right]$, if $c_{i}>\frac{1}{n}$

Proof. We are going to prove that there is $\alpha_{g} \in(0,1)$ such that the second derivative of $v_{i}\left(\alpha_{g}\right)$ equals to zero, for all $i=1,2, \ldots, n$. From the equalities:

$$
\begin{gathered}
e^{t} \mathcal{A}(\alpha) v(\alpha)=\lambda(\alpha), \\
e^{t} \mathcal{A}(\alpha) v(\alpha)=\alpha+(1-\alpha) n c^{t} v(\alpha),
\end{gathered}
$$

we obtain

$$
n \sum_{i=1}^{n} c_{i} v_{i}(\alpha)=\frac{\lambda(\alpha)-\alpha}{1-\alpha} .
$$

By taking the second derivative in the above expression,

$$
n \sum_{i=1}^{n} c_{i} v_{i}^{\prime \prime}(\alpha)=\frac{\lambda^{\prime \prime}(\alpha)(1-\alpha)^{2}+2 \lambda^{\prime}(\alpha)(1-\alpha)+2 \lambda(\alpha)-2}{(1-\alpha)^{3}} .
$$

On the other hand, if we compute the first and the second derivatives in the expression of $\lambda(\alpha)$ obtained in Theorem 1 and we substitute them in the above equation, we get that:

$$
\sum_{i=1}^{n} c_{i} v_{i}^{\prime \prime}(\alpha)=0 \Leftrightarrow F(\alpha)=16 B \alpha^{3}-24 B \alpha^{2}+(9 B-3) \alpha+(1-B)=0,
$$

where $B=\sum_{i<j}\left(c_{i}-c_{j}\right)^{2}$. As $F(0)=1-B>0$, and $F(1)=-2<0$, there is $\alpha_{g} \in(0,1)$ such that $F\left(\alpha_{g}\right)=0$. Moreover, since $F(+\infty)=+\infty, F(\alpha)$ changes its sign after $\alpha=1$, so it has a real root at some $\alpha>1$. With a similar reasoning, since $F(-\infty)=-\infty, F(\alpha)$ has a real root at some $\alpha<0$. Then $\alpha_{g}$ is the unique root in the interval $(0,1)$.

As $r k\left\{w^{i}, i=1,2, \ldots, n-2\right\}$ is a basis of $V^{\perp}$ and $V=\langle e, c\rangle$, it is clear that $r k\left\{c, e, w^{i}, i=1,2, \ldots, n-2\right\}=n$. Then, the linear system

$$
\begin{aligned}
& \text { [1 ] } c^{t} x=0, \\
& \text { [2 ] } e^{t} x=0, \\
& \text { [3 ] }\left(w^{j}\right)^{t} x=0, \forall j=1,2, \ldots, n-2,
\end{aligned}
$$


has a unique solution, $x=0$. As $v^{\prime \prime}\left(\alpha_{g}\right)$ satisfies all conditions, then we obtain $v_{i}^{\prime \prime}\left(\alpha_{g}\right)=0, \quad i=1,2, \ldots, n$. It can be proved (see Remark 2) that $\alpha_{g}<\frac{1}{3}$.

Now, we only need to characterize the components functions $v_{i}(\alpha)$ that change from concave to convex, and vice-versa. As the only zero point for the second derivative is $\alpha_{g}$, we only need to investigate what happens in a point of the interval $\left(\alpha_{g}, 1\right]$.

$$
\begin{gathered}
\lambda(\alpha) v_{k}(\alpha)=\left(e^{k}\right)^{t} \mathcal{A}(\alpha) v(\alpha)=\alpha c_{k}+(1-\alpha) \sum_{i=1}^{n} c_{i} v_{i}(\alpha) . \\
\frac{1}{n} \lambda(\alpha)=\frac{1}{n} \lambda(\alpha) e^{t} v(\alpha)=\frac{1}{n} e^{t} \mathcal{A}(\alpha) v(\alpha)=\alpha \frac{1}{n}+(1-\alpha) \sum_{i=1}^{n} c_{i} v_{i}(\alpha) .
\end{gathered}
$$

By subtracting both equalities, we obtain:

$$
v_{k}(\alpha)=\frac{\alpha}{\lambda(\alpha)}\left(c_{k}-\frac{1}{n}\right)+\frac{1}{n} .
$$

Computing the second derivative at $\hat{\alpha}=\frac{1}{2}$ we get:

$$
\operatorname{sign}\left(v_{k}^{\prime \prime}(\hat{\alpha})\right)=\operatorname{sign}\left(c_{k}-\frac{1}{n}\right)
$$

and the required result holds.

Remark 2. By solving the third order equation that appears in the previous theorem

$$
16 B \alpha^{3}-24 B \alpha^{2}+(9 B-3) \alpha+(1-B)=0
$$

it is not hard to obtain the explicit expression for $\alpha_{g}$ :

$$
\alpha_{g}=1 / 2\left[1-\sqrt{\frac{B+1}{B}} \cos \left(\frac{\arccos \left(\sqrt{\frac{B}{B+1}}\right)+\pi}{3}\right)\right]
$$

Figure 4 shows the graph of this function. An approximation is obtained with the fifth order Taylor polynomial, taken at $B=\frac{1}{3}$. This expression is useful to see that $\frac{1}{3}$ is an upper bound of $\alpha_{g}$.

$$
\alpha_{g} \approx \frac{1}{3}-0.0264 B+0.01335 B^{2}-0.00818 B^{3}+0.00437 B^{4}-0.00130 B^{5} .
$$




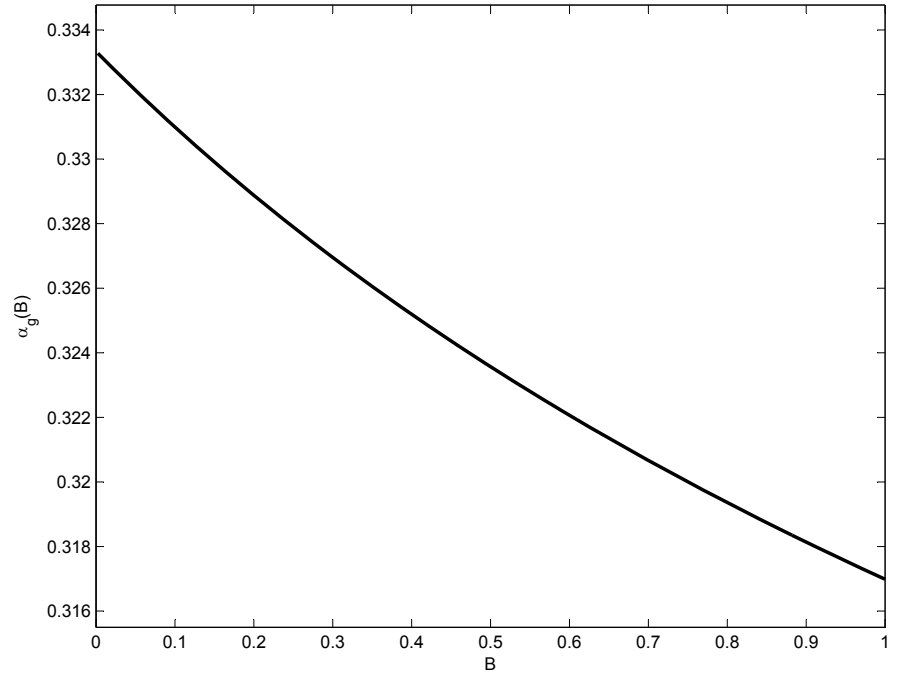

Figure 4: $\alpha_{g}$, as a function of $B=\sum_{i<j}\left(c_{i}-c_{j}\right)^{2}$.

Theorem 4 provides an endogenous way to select a particular value of $\alpha$ in order to select a compromise solution.

Definition 2. Given a cost sharing problem with total cost $M$ and individuals' characteristics $c_{1} \leq c_{2} \leq \ldots \leq c_{n}$, we call $\alpha_{g}$-compromise solution to the allocation defined by

$$
S_{i}\left(\alpha_{g}\right) \equiv M v_{i}\left(\alpha_{g}\right) \quad i=1,2, \ldots, n
$$

where $\alpha_{g}$ is the value obtained in Theorem 4.

Example 3. Consider again the cost sharing problem in Example 1. Then, we first compute the values $B=0.215$ and $\alpha_{g} \approx 0.3282$. Then, the allocation defined by the Perron's eigenvector $v\left(\alpha_{g}\right)$ is

$$
S\left(\alpha_{g}\right)=(16.86,17.49,19.69,20.94,25.02)
$$

which is a compromise between the proposals of egalitarian and the proportional approaches. 
The $\alpha_{g}$-compromise solution sets an abstract compromise based on the mathematical properties of the solution. The meaning of this solution will depend on the model o situation we have. From the mathematical point of view, as the increase of a component in vector $x(\alpha)$ involves decrease in other (others) component, there is no optimal way (for all individuals) to select one of these compromise solutions. If we select the 'inflection' point $\alpha_{g}$ each agent whose participation in the total cost increases with respect to $\alpha\left(c_{i}>\frac{1}{n}\right)$ minimizes her increasing rate at $\alpha_{g}$. Reciprocally, if an agent's allocation decreases with respect to $\alpha\left(c_{i}<\frac{1}{n}\right)$ she maximizes her decreasing rate at $\alpha_{g}$. In this sense the $\alpha_{g}$ point gives a (mathematically) coherent compromise solution.

Remark 3. Nevertheless, this particular solution could yield a "non-admissible" allocation in some situations. For instance, if we have a claims problem (situation [3]) with endowment $M=100$, and agents' claims $c^{t}=$ $[15,18,28.5,34.5,54]$, the characteristics matrix coincides with the one in Example 1, so the $\alpha_{g}$-compromise solution coincide. Then, agent 1 is allocated $x_{1}=16.86$ that is greater than her claim, and this solution is not admissible in claims problems. This trouble is caused because the egalitarian solution itself Egal $=(20,20,20,20,20)$ is not admissible. A similar consideration could be made with respect to situation [4] in which the proposals are usually required to belong to the core of the cooperative game and, in general, the egalitarian solution does not fulfill this condition. We discus this kind of situations in the next section.

\section{A compromise solution as egalitarian as possible}

As mentioned, an egalitarian allocation is no always an admissible solution, in the sense that it is not rational, or does not belong to the core. However, we could be interested in finding an allocation "as egalitarian as possible". As we have shown, our compromise solutions $x(\alpha)$ provide a family of intermediate proposals from the egalitarian $(\alpha=0)$ to the proportional $(\alpha=1)$. Then, we can find suitable values of $\alpha$ in order the allocation $x(\alpha)$ to be admissible and then to find the most egalitarian compromise solution among the admissible ones.

Although the notion "as egalitarian as possible" can be defined in any context, we analyze the problem in two particular situations: inventory cost games and claims problems. 


\subsection{Inventory cost games}

The inventory cost game is defined by associating to every inventory cost situation $\left(N, a,\left(m_{i}\right)_{i \in N}\right),{ }^{5}$ the characteristic function:

$$
c(S)=2 a \sqrt{\sum_{i \in N} m_{i}^{2}}, \text { for } S \subseteq N, S \neq \emptyset ; \quad c(\emptyset)=0 .
$$

Meca et al. (2004) propose a solution in the core of the game defined in a proportional way, that they call SOC-rule:

$$
S O C_{i} \equiv \frac{c^{2}(i)}{\sum_{j=1}^{n} c^{2}(j)} c(N) \quad i \in N
$$

This is a kind of proportional solution, where proportionality is taken with respect to the characteristics $c_{i}=c^{2}(i)$.

Nevertheless, the egalitarian allocation

$$
x_{i}=\frac{c(N)}{n} \quad i \in N
$$

has not been used in this context since, in general, it does no belong to the core. In our family of compromise solutions we may find the minimum value of $\alpha$ such that $x(\alpha)$ belongs to the core (that is, we may obtain the most egalitarian compromise solution in the core of the inventory cost game). This solution is obtained by finding:

$\alpha_{e q}=\min \left\{\alpha \in[0,1]: \sum_{i \in N} x_{i}(\alpha)=c(N), \sum_{i \in S} x_{i}(\alpha) \leq c(S)\right.$, for all $\left.S \subset N\right\}$

This minimal value always exist since $x(1)$ coincides with the proportional solution (the $S O C$-rule) and $x(\alpha)$ is continuous with respect to $\alpha$.

In order to illustrate the range of values of $\alpha$ such that $x(\alpha)$ gives an allocation in the core of the game, we use the following example taken from Meca et al. (2004) slightly modified. ${ }^{6}$

${ }^{5} N=\{1,2, \ldots, n\}$ denotes the set of agents, $m_{i}$ is the optimal number of orders per time unit for agent $i$ if she does not cooperate, and $a$ is the fixed cost of an order (Fiestras-Janeiro et al. (2011)).

${ }^{6}$ In the original example the egalitarian allocation is in the core. 
We consider three airline companies, L1, L2, L3 that operate in the same country. [...] Over time, each firm has learned how much taillights are used on the average in a year: $L 1$ needs 50 taillights per year, L2 needs 400, and L3 needs 500. The holding cost to store one light for one year is, respectively, 11, 10 and 9.6 dollars. The cost of placing an order for taillights equals 600 dollars.

This situation defines the inventory cost game with function:

$$
\begin{aligned}
& c(\{1\})=812.4 ; \quad c(\{2\})=2190.89 ; \quad c(\{3\})=2400 ; \\
& c(\{1,2\})=2336.7 ; \quad c(\{1,3\})=2533.6 ; \quad c(\{2,3\})=3249.6 \\
& c(\{1,2,3\})=3349.6 \text {. }
\end{aligned}
$$

The $S O C$-rule allocates the total cost $c(N)$ proportionally to the coefficients $c_{i}=c^{2}(\{i\})$, so the normalized characteristics matrix is:

$$
A=\left(\begin{array}{lll}
0.0588 & 0.4278 & 0.5134 \\
0.0588 & 0.4278 & 0.5134 \\
0.0588 & 0.4278 & 0.5134
\end{array}\right)
$$

Then, the allocation $x(\alpha)$ is in the core of the game for all $\alpha \in[0,1]$, such that $\alpha \geq \alpha_{e q} \approx 0.35545$, and this value determines the most egalitarian compromise solution in the core. The allocation proposed by this compromise solution is $x_{e q}=[812.4,1221.2,1316]$. The $S O C$-rule proposes the allocation $S O C=[197,1433,1719.6]$, that coincides with the compromise solution for $\alpha=1$. Note that, being the compromise solution as egalitarian as possible, agent 1 is allocated their maximum rational amount, $c(\{1\})$. Proposals $x(\alpha)$ with $\alpha_{e q}<\alpha<1$ provide intermediate proposals between $x_{e q}$ and the $S O C$ solution.

\subsection{Claims problems}

We now consider a claims problem defined by the pair $(M, r)$, where $M$ is the endowment and $r^{t}=\left[r_{1}, r_{2}, \ldots, r_{n}\right]$ are the claims of the agents. The situation is a conflicting claims problem (bankruptcy) when $R=\sum_{i=1}^{n} r_{i}>$ $M$. As noticed in Remark 3, the egalitarian proposal

$$
\text { Egal }_{i}=\frac{M}{n} \quad i=1,2, \ldots, n
$$


is not in general admissible (that, in this context, means that no agent should receive more than her claim). In this case, we may find the value of $\alpha$ that delimits the admissible compromise solutions.

Theorem 5. Let $A$ be a characteristics matrix with row vector $c^{t}$, and $v(\alpha)$ the normalized Perron's eigenvector associated to the Levinger's transformation $\mathcal{A}(\alpha)=\alpha A^{t}+(1-\alpha) A$. Let $\kappa \in \mathbb{R}, \kappa>1$. Then, there is $\alpha_{\text {eq }} \in[0,1]$ such that:

$$
v(\alpha) \leqq \kappa c \Leftrightarrow \alpha \geq \alpha_{e q}
$$

Proof. If we compute $\mathcal{A}(\alpha) v(\alpha)$,

$$
\mathcal{A}(\alpha) v(\alpha)=\alpha A^{t} v(\alpha)+(1-\alpha) A v(\alpha)=\alpha c+(1-\alpha)\left(\sum_{i=1}^{n} c_{i} v_{i}(\alpha)\right) e
$$

and, from Theorems 1 and 3, we know that

$$
\sum_{i=1}^{n} c_{i} v_{i}(\alpha)=\frac{\alpha \sum_{i=1}^{n} c_{i}^{2}}{\lambda(\alpha)-1+\alpha}, \quad \lambda(\alpha)=\frac{1+\sqrt{1+4 \alpha(1-\alpha) B}}{2} .
$$

Then, we have the inequality

$$
v(\alpha)=\frac{1}{\lambda(\alpha)} \mathcal{A}(\alpha) v(\alpha)=\frac{1}{\lambda(\alpha)}\left(\alpha c+\frac{\alpha(1-\alpha) \sum_{i=1}^{n} c_{i}^{2}}{\lambda(\alpha)-1+\alpha} e\right) \leqq \kappa c
$$

which is equivalent to

$$
\frac{\alpha(1-\alpha) \sum_{i=1}^{n} c_{i}^{2}}{\lambda(\alpha)-1+\alpha} e \leqq(\kappa \lambda(\alpha)-\alpha) c .
$$

Note that the left hand of this inequality is constant for all $i$. So, as the entries in $c$ are increasingly ordered, then (1) only requires to be checked for $i=1$,

$$
\frac{\alpha(1-\alpha) \sum_{i=1}^{n} c_{i}^{2}}{\lambda(\alpha)-1+\alpha} \leqq(\kappa \lambda(\alpha)-\alpha) c_{1},
$$

that obviously holds for $\alpha=1$. The following value provides the equality

$$
\alpha_{e q}=\frac{\kappa^{2} B^{2} c_{1}^{2}-2 \kappa B C c_{1}+\kappa(\kappa+1) B c_{1}^{2}+C^{2}-(\kappa+1) C c_{1}+\kappa c_{1}^{2}}{\kappa^{2} B^{2} c_{1}^{2}-2 \kappa B C c_{1}+\left(\kappa^{2}+1\right) B c_{1}^{2}+C^{2}-2 C c_{1}+c_{1}^{2}}
$$


where

$$
B=\sum_{i<j}\left(c_{i}-c_{j}\right)^{2}, \quad C=\sum_{i=1}^{n} c_{i}^{2} .
$$

In order to prove that for any $\alpha \in\left[\alpha_{e q}, 1\right]$ the inequality (2) holds, it is sufficient to observe that the relevant condition is $v_{1}(\alpha) \leq \kappa c_{1}$ and that from Theorem $3 v_{1}(\alpha)$ is a decreasing function. So, $\alpha>\alpha_{e q}, \alpha \in[0,1]$, implies $v_{1}(\alpha) \leq v_{1}\left(\alpha_{e q}\right) \leq \kappa c_{1}$.

As a consequence we obtain the following result for claims problems.

Corollary 2. Let $(M, r)$, a claims problem and $A$ the characteristics matrix with $c_{i}=\frac{r_{i}}{\sum_{j=1}^{n} r_{j}}$. Then there is $\alpha_{e q} \in[0,1]$ such that for all $\alpha \in\left[\alpha_{e q}, 1\right]$ $x(\alpha)$ is an admissible allocation, that is

$$
x(\alpha) \leqq r .
$$

Proof. The compromise solution is $x(\alpha)=M v(\alpha)$. Then, we only need to apply Theorem 5 by considering

$$
\kappa=\frac{\sum_{i=1}^{n} r_{i}}{M}
$$

that is greater than 1.

Moreover, as in the case of Subsection 4.1, $\alpha_{e q}$ provides the most egalitarian compromise solution among the admissible ones.

\section{Acknowledgments}

We are particularly grateful to two anonymous referees and the Editor for many valuable comments and suggestions that have led to a substantial improvement in the manuscript. Financial support from Spanish Ministry of Economy and Competitiveness under Project ECO2013-43119 is gratefully acknowledged. SilvaReus also acknowledges financial support from the Generalitat Valenciana under project PROMETEO/2009/068. 


\section{References}

Berman, A., Plemmons, R., 1994. Nonnegative Matrices in the Mathematical Sciences. Classics in Applied Mathematics, SIAM, Philadelphia, PA.

Fiedler, M., 1995. Numerical range of matrices and Levinger's theorem. Linear Algebra and App. (220), 171-180.

Fiestras-Janeiro, M., García-Jurado, I., Meca, A., Mosquera, M., 2011. Cooperative game theory and inventory management. European Journal of Operational Research 210 (3), 459 - 466.

Giménez-Gómez, J. M., Peris, J. E., 2014. A proportional approach to claims problems with a guaranteed minimum. European Journal of Operational Research 232 (1), 109-116.

Karsten, F., Basten, R. J., 2014. Pooling of spare parts between multiple users: How to share the benefits? European Journal of Operational Research 233 (1), 94-104.

Levinger, B., 1970. An inequality for nonnegative matrices. Notices Amer. Math. Soc. (17), 260.

Meca, A., Timmer, J., García-Jurado, I., Borm, P., 2004. Inventory games. European Journal of Operational Research 156 (1), 127-139.

Moulin, H., 1988. Axioms of Cooperative Decision Making. Cambridge University Press, Cambridge.

O'Neill, B., 1982. A problem of rights arbitration from the Talmud. Mathematical Social Sciences 2 (4), 345-371.

Perron, O., 1907. Zur theorie der matrizen. Math. Ann. (64), 248-263.

Turkensteen, M., Klose, A., 2012. Demand dispersion and logistics costs in one-to-many distribution systems. European Journal of Operational Research 223 (2), 499-507. 